\title{
Estudo cefalométrico da correlação da anatomia da base craniana com o padrão facial e as bases apicais
}

Marcelo Calvo de Araújo*, Ana Carla Raphaelli Nahás**, Flavio Augusto Cotrim-Ferreira***, Paulo Eduardo Guedes Carvalho***

\section{Resumo}

Objetivos: este estudo transversal foi realizado com o objetivo de avaliar cefalometricamente a correlação da anatomia da base craniana com o padrão facial e as bases apicais. Metodologia: foram utilizadas 88 telerradiografias de norma lateral de jovens leucodermas brasileiros com média de idade de 10,3 anos. Utilizou-se o índice VERT de Ricketts para a determinação do padrão facial, distribuindo a amostra em: 37 para o grupo M (mesofaciais), 34 para o grupo $\mathrm{D}$ (dolicofaciais) e 17 para o grupo B (braquifaciais). Realizaram-se, manualmente: o desenho anatômico, a demarcação de pontos, o traçado de linhas e planos e a aferição de medidas lineares e angulares. As medidas da base do crânio utilizadas foram S-N, N.S.Ba e S-N.Po-Or, e as medidas das bases apicais foram S.N.A, S.N.B e A.N.B. Resultados e Conclusões: concluiu-se que, na correlação entre a base craniana e o padrão facial, houve significância entre a variável N.S.Ba e o índice VERT. Na correlação entre a base craniana e as bases apicais, houve significância entre N.S.Ba e as variáveis S.N.A e S.N.B, e entre S-N.Po-Or e as variáveis S.N.A e S.N.B.

Palavras-chave: Padrão facial. Base craniana. Cefalometria.

\section{INTRODUÇÃO}

Dentre os vários métodos utilizados para a realização do planejamento ortodôntico, a análise obtida sobre a telerradiografia é importante para a determinação do diagnóstico e dos objetivos finais do tratamento. A cefalometria constitui um valioso exame complementar, porém é imprescindível conhecê-la, adequada e corretamente, para a realização de um ótimo diagnóstico e plano de tratamento.

Ultimamente, tem-se visto que as médias cefalométricas tradicionais, utilizadas nos diversos traçados cefalométricos, não podem ser aplicadas de forma genérica. São necessárias individualizações - considerando o gênero, a idade, a raça, o padrão facial, o padrão respiratório, além do senso clínico - para avaliar o padrão de beleza requerido pelo paciente e pela sociedade local. Diversos

* Coordenador do curso de especialização Centro de Estudos Smile - núcleo Funorte - Niterói/RJ. Professor do curso de especialização ABOM-

RJ.

Mestre e Doutora em Ortodontia FOB-USP. Professora Associada do Curso de Mestrado Profissionalizante em Ortodontia da Universidade Cidade de São Paulo - UNICID.

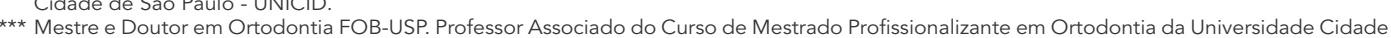
de São Paulo - UNICID. 
pesquisadores ${ }^{1,14,20}$ concluíram que a maioria das normas cefalométricas varia de maneira significante, se comparados os diversos padrões faciais (mesofacial, braquifacial e dolicofacial). Em vista disso, uma interpretação mais personalizada da cefalometria deve tornar-se regra.

Vários estudos ${ }^{3,21,25,26}$ foram realizados com o intuito de correlacionar a anatomia da base craniana com as bases apicais, cujos resultados obtidos demonstraram haver uma correlação significativa. Indivíduos com ângulo N.S.Ba aumentado tendem a apresentar S.N.A e S.N.B. menores, e vice-versa. Poucos estudos ${ }^{31}$ foram efetuados utilizando o plano de Frankfurt como referência para medir a inclinação da base anterior do crânio (S-N) em trabalhos comparativos de base craniana com bases apicais. Outro fator importante comprovado em inúmeros estudos ${ }^{2,13,17,18,23}$ é a definição e estabilidade da deflexão da base craniana por volta de 3 anos de idade, podendo, assim, se tornar um indicativo precoce do padrão de face, que irá se consolidar mais adiante. Delaire ${ }^{11}$ afirmou ser a base do crânio o alicerce sobre o qual se edifica a face humana, por isso sua anatomia influencia a arquitetura da face. Vale ressaltar que um dos fatores importantes na definição do diagnóstico e da mecanoterapia a ser utilizada durante o tratamento é o padrão facial do indivíduo. A aquisição desta informação na fase inicial de desenvolvimento da criança, através da correlação proposta neste trabalho, se torna mais uma ferramenta no arsenal de dados iniciais para o planejamento da terapia a ser utilizada.

\section{PROPOSIÇÃO}

Este estudo se propôs a avaliar:

1) A correlação da anatomia da base craniana com o padrão facial, abordando as correlações das seguintes grandezas: correlação do comprimento da base anterior do crânio (S-N), correlação do ângulo formado pela base anterior do crânio e o plano de Frankfurt (S-N.Po-Or) e a correlação da deflexão da base do crânio (N.S.Ba) com o índice indicativo do padrão facial (VERT).

2) A correlação da anatomia da base craniana com as bases apicais, abordando as correlações das seguintes grandezas: correlação do comprimento da base anterior do crânio (S-N), correlação do ângulo formado pela base anterior do crânio (S-N) e o plano de Frankfurt (S-N.Po-Or) e correlação da deflexão da base do crânio (N.S.Ba) com as bases apicais, analisadas pelos ângulos S.N.A, S.N.B e A.N.B.

\section{MATERIAL E MÉTODOS}

Esse trabalho respeitou todos os requisitos exigidos pelo comitê de ética em pesquisa da Universidade Cidade de São Paulo para a sua realização, obtendo aprovação.

\section{Composição, critérios de seleção e divisão da amostra}

A amostra foi constituída de 88 telerradiografias de jovens brasileiros leucodermas, descendentes de portugueses, espanhóis ou italianos, de ambos os gêneros, na faixa etária dos 7 aos 13 anos, com média de idade de 10,3 anos, apresentando má oclusão de Classes I e II de Angle, com documentação ortodôntica obtida no arquivo da clínica de Ortodontia e Odontopediatria da Universidade Cidade de São Paulo - UNICID.

Foram empregadas, neste trabalho, exclusivamente as telerradiografias em norma lateral de pacientes que foram radiografados antes do tratamento ortodôntico fornecido pela disciplina de Ortodontia da UNICID. Foram excluídos pacientes com tratamento ortodôntico prévio, pacientes portadores de alterações patológicas como disostose cleidocraniana, fenda palatina, síndrome de Down, etc. Estas alterações patológicas apresentam alterações na morfologia de base craniana. Foram, também, excluídas telerradiografias que não apresentavam boa nitidez para a execução do cefalograma. A partir destes critérios, foram analisadas 120 telerradiografias e 88 apresentaram os requisitos para compor a amostra. 
Na presente pesquisa, os indivíduos foram distribuídos em três grupos, da seguinte forma: 17 braquifaciais (grupo B), 37 mesofaciais (grupo M) e 34 dolicofaciais (grupo D). Foi utilizado o índice VERT de Ricketts para a determinação do padrão facial.

\section{MÉTODOS}

\section{Elaboração do cefalograma}

Todos os passos para obtenção do cefalograma foram realizados pelo pesquisador, de modo manual, e estão descritos a seguir.

Desenho anatômico e determinação dos pontos cefalométricos

Sobre cada uma das 88 telerradiografias selecionadas para compor esta amostra, fixou-se, com fita adesiva transparente, uma folha de papel Ultraphan (GAC) de formato retangular, com espessura de $0,07 \mathrm{~mm}$ e dimensões de $17,5 \times 17,5$ centímetros. Em sala obscurecida, posicionou-se o conjunto sobre um negatoscópio, adaptando ao

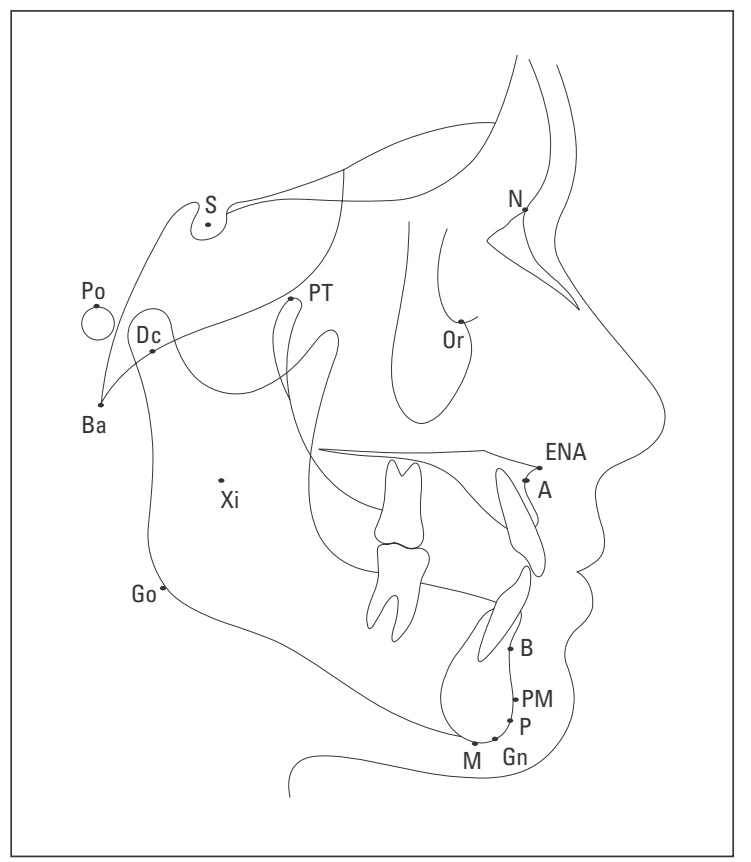

FIGURA 1 - Desenho anatômico e pontos cefalométricos. redor uma máscara de cartolina preta, de modo a eliminar ao máximo a luminosidade lateral proveniente do negatoscópio. Copiou-se da radiografia, com lapiseira provida de grafite $0,3 \mathrm{~mm}$, as estruturas anatômicas, preconizadas pela análise de Ricketts e, posteriormente, foi feita a demarcação dos pontos cefalométricos (Fig. 1) necessários às aferições das medidas propostas neste estudo.

\section{Mensuração das grandezas cefalométricas}

\section{a) Grandezas para determinar o índice VERT}

O VERT é obtido a partir de cinco grandezas cefalométricas (Fig. 2): (1) altura facial inferior; (2) profundidade facial; (3) ângulo do eixo facial; (4) ângulo do plano mandibular e (5) arco mandibular.

Para atingir o resultado do VERT foram seguidos os seguintes passos:

- foram medidos os valores angulares das cinco grandezas relacionadas acima. Os valores foram medidos e anotados manualmente.

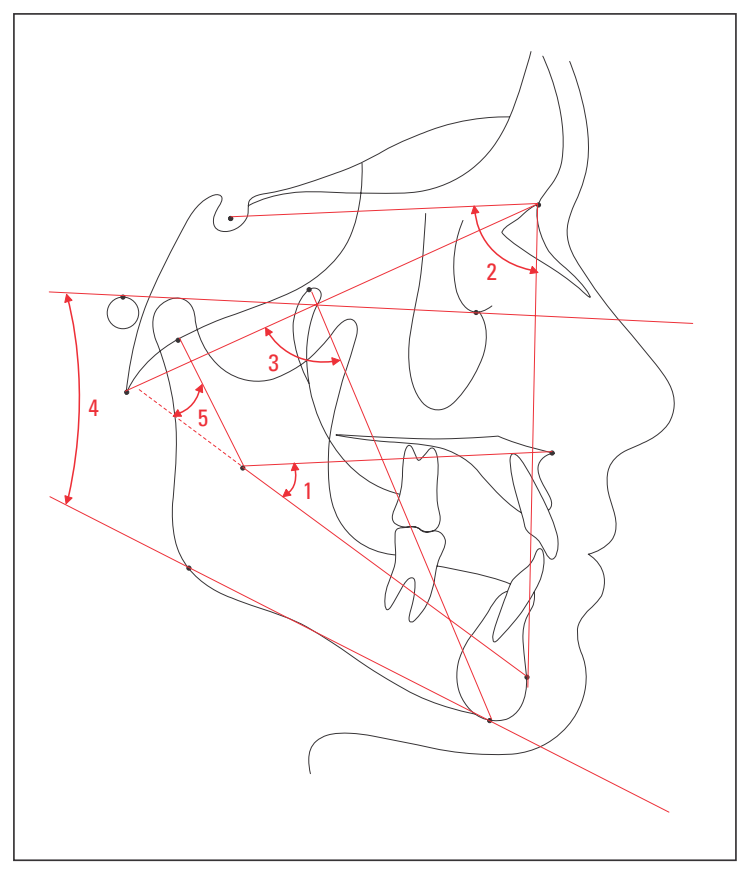

FIGURA 2 - Grandezas do índice VERT. 
- foi estabelecida a diferença, em graus, entre o valor obtido no paciente e a norma clínica individualizada para cada idade. Ricketts ${ }^{25}$, pelo estudo de centenas de exames cefalométricos, determinou um valor normal para cada ângulo e suas variações com a idade ${ }^{15}$.

- foi dividida a diferença em graus pelo desvio clínico, atentando para o fato de que o resultado deve ter sinal positivo quando o valor angular do paciente indicar uma direção mais horizontal que a norma clínica, e valor negativo quando representar uma tendência mais vertical.

Os resultados obtidos acima, para cada um dos cinco ângulos, foram somados e divididos por 5 . O valor alcançado é denominado VERT.

Quando o valor do VERT situou-se no intervalo entre $-0,5$ e $+0,5$ o indivíduo foi classificado como mesofacial. Valores do VERT maiores que 0,5 apontaram os jovens braquifaciais e valores menores que $-0,5$ indicaram os pacientes cujo padrão facial foi classificado como dolicofacial. A tabela 1 exprime as relações entre o VERT e os padrões faciais.

\section{b) Grandezas para determinar anatomia da base craniana}

Foram traçadas as seguintes grandezas cefalométricas para determinar a base craniana (Fig. 3):

1) ângulo N.S.Ba - formado pela intersecção da linha S-N com a linha S-Ba, que representa a angulação da base craniana.

2) ângulo S-N.Po-Or - formado pela intersecção da linha S-N com a linha Po-Or.

3) distância S-N - medida linear da distância em milimetros, entre os pontos S (Sela) e Násio $(\mathrm{N})$, a qual representa o comprimento da base anterior do crânio.

\section{c) Grandezas para determinar as bases apicais}

Foram traçadas as seguintes grandezas cefalométricas para determinar as bases apicais (Fig. 4):

1) ângulo S.N.B - formado pela intersecção da linha S-N com a linha N-B.
Tabela 1 - Relação entre VERT e padrão facial.

\begin{tabular}{ccc}
\hline dolicofacial & mesofacial & braquifacial \\
\hline VERT $<-0,5$ & $-0,5 \leq$ VERT $\geq 0,5$ & VERT $>0,5$ \\
\hline
\end{tabular}

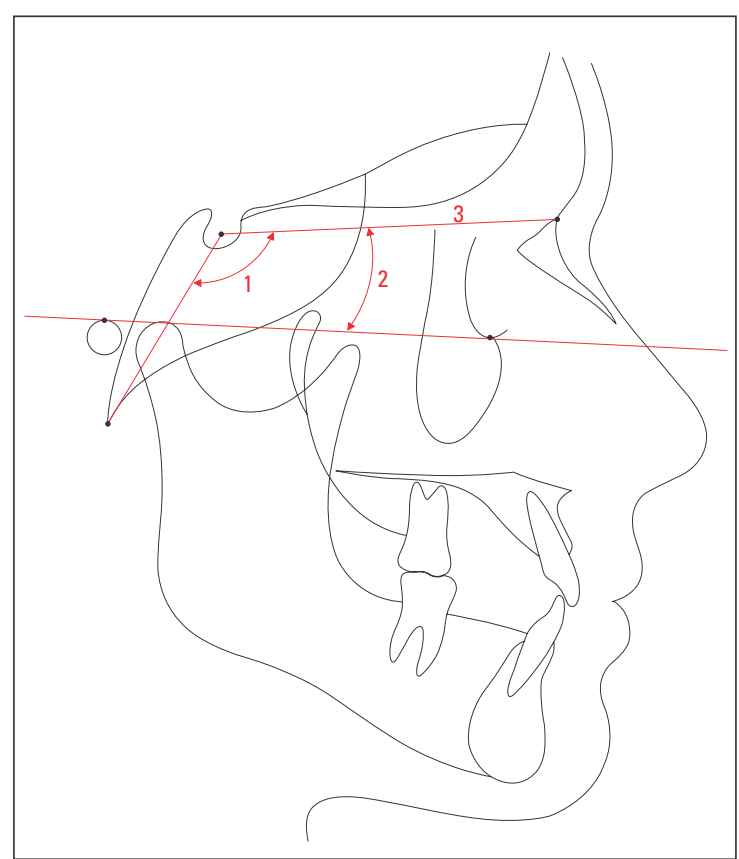

FIGURA 3 - Grandezas da base craniana.

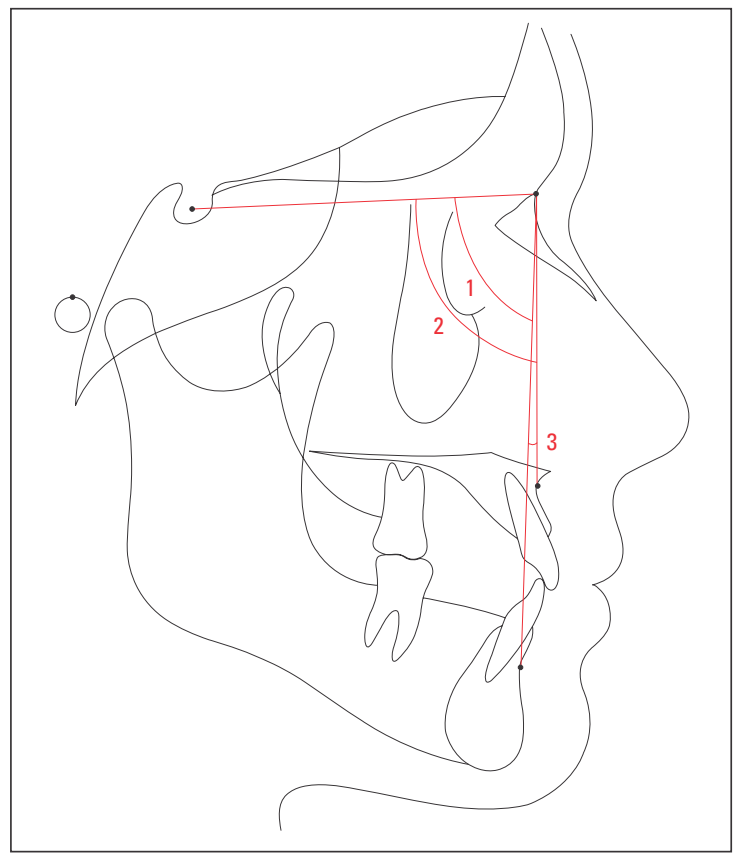

FIGURA 4 - Grandezas das bases apicais. 
2) ângulo S.N.A - formado pela intersecção da linha S-N com a linha N-A.

3) ângulo A.N.B - formado pela intersecção da linha N-A com a linha N-B.

\section{Erro do método}

Com o objetivo de determinar a margem de erro intra-examinador, 30 das 88 telerradiografias foram retraçadas após um intervalo de 10 dias, obedecendo aos mesmos procedimentos realizados no primeiro traçado. Os resultados dos dois traçados foram comparados pelo teste $\mathrm{t}$ de Student, após terem sido feitos a média e o desviopadrão em cada variável estudada, nos dois traçados. Não foi observada a significância estatística do erro, aumentado, assim, a confiabilidade destes achados.

\section{Metodologia aplicada para as análises estatísticas}

Para serem avaliadas as correlações da anatomia da base do crânio com o padrão facial e as bases apicais, foram obtidos a média e o desviopadrão das variáveis e utilizou-se o coeficiente de correlação de Pearson (r) para cada correlação. Realizou-se, também, o teste $t$ de Student de cada correlação, obtendo o valor de p, t e o nível de significância que determina quais correlações foram estatisticamente significantes. Neste trabalho foram consideradas significativas as diferenças para o nível de significância de 0,05 (5\%) (p<0,05).

\section{RESULTADOS}

Médias e desvios-padrão das variáveis da anatomia da base do crânio e das bases apicais dos três

Tabela 2 - Valores médios e desvios-padrão encontrados para as variáveis da base do crânio no grupo B (braquifaciais), no grupo M (mesofaciais), no grupo D (dolicofaciais) e no grupo T (total).

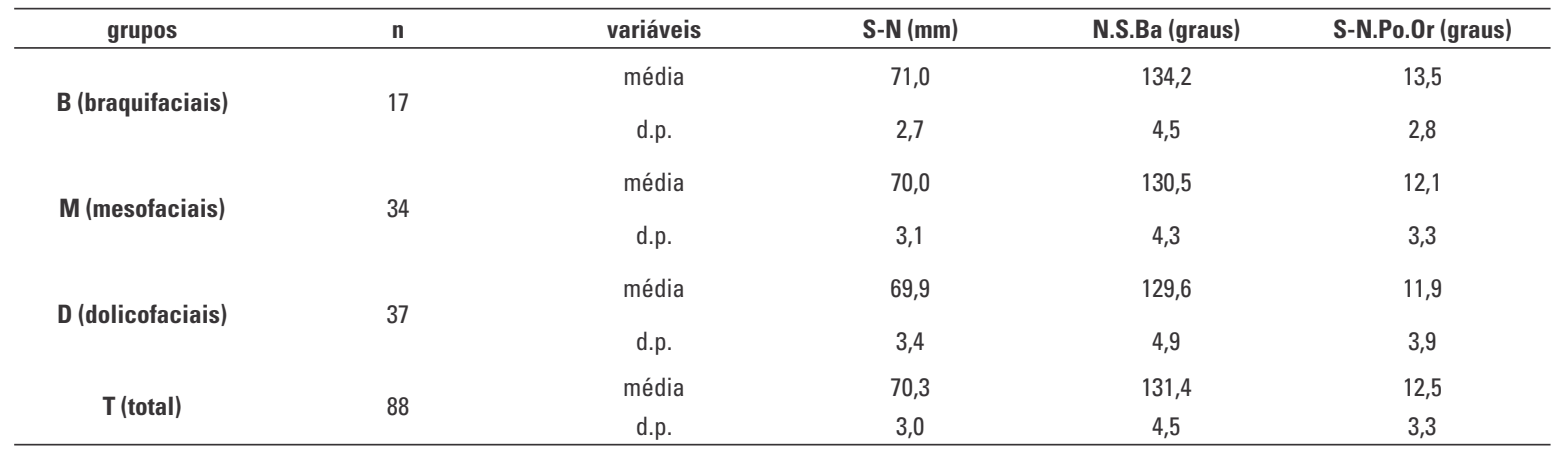

Tabela 3 - Valores médios e desvios-padrão encontrados para variáveis das bases apicais no grupo B (braquifaciais), no grupo $\mathrm{M}$ (mesofaciais), no grupo $\mathrm{D}$ (dolicofaciais) e no grupo T (total).

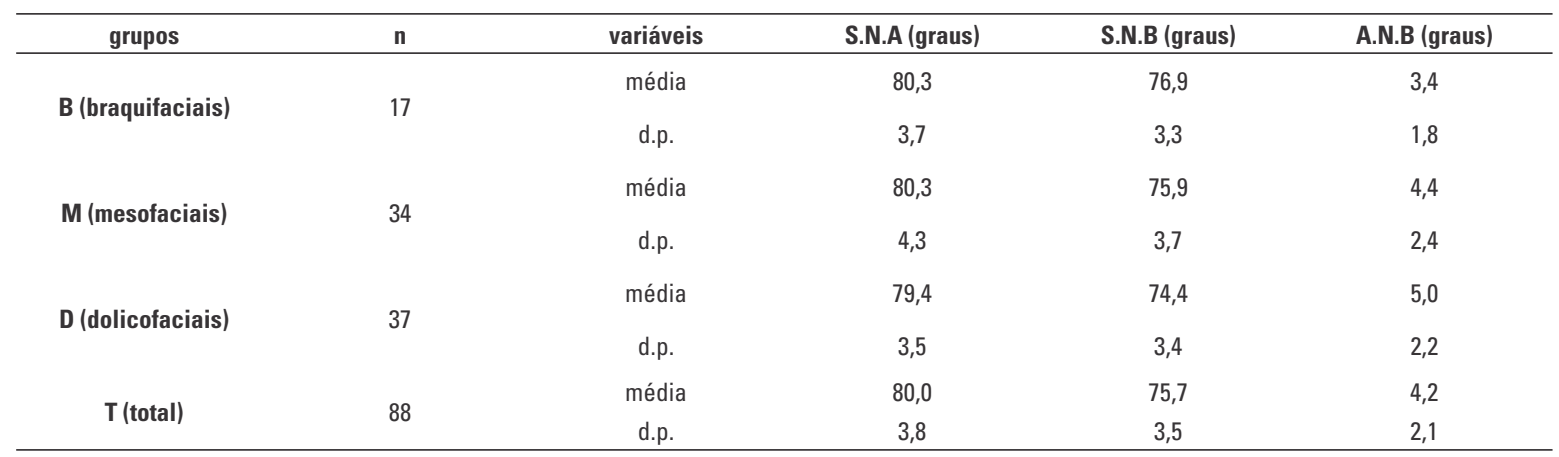


grupos estudados e do grupo total.

As tabelas 2 e 3 exibem as médias e os desviospadrão para todas as variáveis cefalométricas estudadas, referentes à anatomia da base do crânio e bases apicais para o grupo B (braquifaciais), para o grupo M (mesofaciais), para o grupo D (dolicofaciais) e para o grupo T (total). Verifica-se que os braquifaciais mostraram valores absolutos maiores em todas as variáveis da base do crânio, em relação aos demais grupos. Os mesofaciais apresentaram valores intermediários e os dolicofaciais apresentaram valores menores.

\section{Correlação das variáveis da base do crânio com o índice indicativo do padrão facial (VERT) e as bases apicais}

As correlações das variáveis representativas da base craniana com o índice VERT (Tab. 4) e com as bases apicais (Tab. 5) foram realizadas por

Tabela 4 - Valores de r, t, p e nível de significância das correlações das variáveis da base do crânio com o índice VERT.

\begin{tabular}{|c|c|c|c|}
\hline & \multicolumn{3}{|c|}{ variáveis da base do crânio } \\
\hline & S-N & N.S.Ba & S-N.Po-Or \\
\hline & \multicolumn{3}{|c|}{ índice indicativo do padrão facial } \\
\hline & VERT & VERT & VERT \\
\hline n & 88 & 88 & 88 \\
\hline $\mathbf{r}$ & 0,11 & 0,23 & 0,02 \\
\hline $\mathbf{t}$ & 0,994 & 2,207 & 0,142 \\
\hline $\mathbf{p}$ & 0,323 & 0,030 & 0,888 \\
\hline Sig. & n.s. & 0,05 & n.s. \\
\hline
\end{tabular}

meio do coeficiente de correlação de Pearson (r), realizando o teste estatístico de cada correlação, obtendo o valor de r, t, p e o nível de significância que determina quais correlações foram estatisticamente significantes.

\section{DISCUSSÃO}

Diversas análises ${ }^{18,28,29}$ foram descritas na literatura com o objetivo de auxiliar no diagnóstico ortodôntico. No início, as normas cefalométricas constituíam verdadeiras metas a serem alcançadas. Pouco a pouco, os ortodontistas passaram a considerar o exame cefalométrico com mais cautela, tendo em vista a realidade das amplas variações e compensações individuais, o que foi perfeitamente relatado por Enlow, Kuroda e Lewis ${ }^{12}$, em 1971.

\section{Correlação da anatomia da base craniana com o padrão facial}

Christie $^{8}$ constatou que a maioria das normas varia significantemente de acordo com o padrão facial. Cotrim-Ferreira ${ }^{9,10}$ encontrou a mesma tendência apresentada na presente pesquisa, em relação ao ângulo da base do crânio e à variável S-N (maiores em braquifaciais e menores em dolicofaciais). O valor médio de N.S.Ba $\left(131,4^{\circ}\right)$ encontrado na amostra atual ficou muito próximo de valores médios encontrados $\left(130^{\circ}\right)$ em outros estudos $2,4,9,10,22,24$.

Como pode ser visto na tabela 4 , por meio do teste $t$ de Student das correlações realizadas, na correlação das variáveis da base do crânio com o índice

Tabela 5 - Valores de r, t, p e nível de significância das correlações das variáveis de base do crânio com as variáveis das bases apicais.

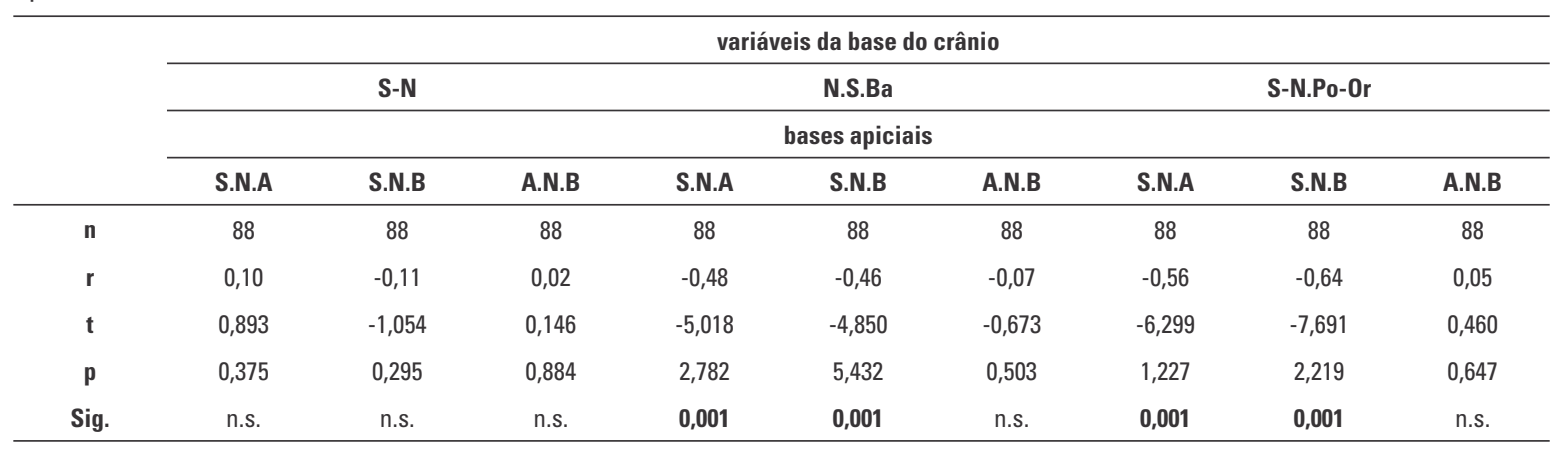


indicativo do padrão facial (VERT), houve correlação positiva estatisticamente significante apenas para a variável representativa do ângulo da base do crânio (N.S.Ba). Esta correlação positiva (quanto maior N.S.Ba maior o valor VERT) demonstrou a importância da anatomia da base craniana na conformação facial, como bem apontado por Brodie 4 . Este autor relatou que alterações ocorridas na base craniana manifestar-se-iam na porção facial à qual a base está ligada. As variáveis S-N e S-N.Po-Or não apresentaram correlação estatisticamente significante. A base anterior do crânio, representada por $\mathrm{S}-\mathrm{N}$, isoladamente parece não influenciar o padrão da face, e sim a base craniana como um todo; com a base anterior e posterior mantendo uma certa proporcionalidade entre si, como demonstrado por Cotrim-Ferreira ${ }^{9}$, representando, assim, um verdadeiro alicerce para edificação da face.

Sakima ${ }^{27}$ relatou a não observância da correlação padrão facial com ângulo da base do crânio. Porém, neste estudo foram utilizados apenas pacientes Classe II, divisão 1 . Ramos ${ }^{26}$ não encontrou correlação significante entre N.S.Ba e o ângulo indicativo da divergência facial (S-N.Go-Gn). Já Sielaffe ${ }^{28}$, Bhat e Enlow ${ }^{1}$ constataram que os indivíduos de padrão vertical tinham tendência a ter o ângulo N.S.Ba aumentado, contrariamente aos braquifaciais. Neste trabalho não foi utilizado o índice VERT para a separação dos grupos faciais e a amostra tinha faixa etária maior e origem étnica diferente da utilizada nesta pesquisa. Metzdor $\mathrm{f}^{20}$ constatou que os indivíduos de padrão vertical tinham tendência a ter o ângulo N.S.Ba diminuído, contrariamente aos braquifaciais, vindo, assim, de encontro aos nossos achados. Para isto, Metzdorf ${ }^{20}$ utilizou o FMA para a distinção dos padrões faciais. Cotrim-Ferreira ${ }^{9,10}$, trabalhando com uma amostra de origem étnica muito semelhante à utilizada neste estudo e fazendo uso da mesma metodologia para a separação dos grupos faciais, encontrou resultados semelhantes aos desta pesquisa, ao detectar um menor ângulo N.S.Ba nos portadores de crescimento vertical e um maior em portadores de crescimento horizontal.

\section{Correlação da anatomia da base craniana com as bases apicais}

Para a verificação da correlação entre as variáveis da base do crânio e as variáveis das bases apicais utilizou-se o coeficiente de correlação de Pearson na amostra total. Foi realizada a correlação de cada variável da base do crânio com as variáveis das bases apicais e também foi feito o teste $t$ de Student de todas as correlações. Os resultados dos testes estatísticos das correlações estão demonstrados na tabela 5 . A variável $\mathrm{S}-\mathrm{N}$ não apresentou correlação com nenhuma variável das bases apicais estudadas, mostrando haver certa relação de proporcionalidade linear entre o comprimento da base anterior do crânio, maxila e mandíbula, com conseqüente relação entre a localização do ponto $\mathrm{N}$ e os pontos $\mathrm{A}$ e $\mathrm{B}$, de maneira que o comprimento da base anterior do crânio não se correlaciona com as medidas angulares das bases apicais estudadas. Já as variáveis N.S.Ba e S-N.Po-Or mostraram correlação negativa estatisticamente significante com as variáveis S.N.A e S.N.B. Quanto maior o valor de N.S.Ba menor o valor de S.N.A e S.N.B, e vice-versa. Estes resultados vieram de encontro com resultados obtidos por outros autores ${ }^{3,26,27}$. Esta relação mostra a importância do posicionamento vertical do ponto $\mathrm{N}$, ditando assim a inclinação da base anterior do crânio e conseqüente valor dos ângulos S.N.A e S.N.B. Rezk ${ }^{21}$ observou que a linha sela-násio poderia ser alterada por um movimento vertical do ponto $\mathrm{N}$, o que deve ser verificado, visto ser uma linha de referência cefalométrica. Já a variável A.N.B não se correlacionou com nenhuma variável da base do crânio, mostrando que as rotações das bases ocorrem em conjunto, como demonstrado por Jarvinem ${ }^{16}$ em amostra com diferentes padrões faciais, mantendo, assim, as relações entre as bases inalteradas.

Carreiro ${ }^{7}$, em trabalho realizado em 1996, não encontrou relação entre o ângulo da base do crânio (N.S.Ba) e as bases apicais, porém este autor utilizou a linha $\mathrm{N}$-perpendicular da análise de 
McNamara ${ }^{19}$ para avaliar o posicionamento das bases apicais. Este resultado, comparado ao deste trabalho, mostra que a correlação do ângulo da base do crânio com as bases apicais está ligada à inclinação da base anterior do crânio (S-N) e é significante quando as bases apicais são representadas pelos ângulos S.N.A e S.N.B, já não apresentando a mesma significância quando representados pela distância dos pontos A e B à linha $\mathrm{N}$-perpendicular.

Os resultados dos valores das variáveis das bases apicais encontrados neste estudo vieram de encontro com os de outros trabalhos ${ }^{6,14}$ que mostraram S.N.A e S.N.B menores no grupo de verticais e maiores no grupo de horizontais.

O trabalho de Cardoso ${ }^{6}$ apresentou uma amostra de origem étnica muita semelhante à utilizada nesta pesquisa, mas utilizou como método de separação dos padrões faciais a avaliação da morfologia da face por meio de fotografias, observando: terço inferior e médio da face, linha queixo-pescoço reduzida, ângulo queixo-pescoço fechado, ausência de selamento labial passivo e excesso de exposição de incisivos superiores com o lábio em repouso, sem levar em consideração relações ósseas e oclusais. Os autores tiveram resultados absolutos para o grupo de padrão vertical de S.N.A $\left(79,9^{\circ}\right)$, S.N.B $\left(74,0^{\circ}\right)$ e A.N.B $\left(5,8^{\circ}\right)$ muito próximos aos valores encontrados neste trabalho, que foram de S.N.A de $79,4^{\circ}$, S.N.B de $74,4^{\circ}$ e A.N.B de $5,0^{\circ}$ e que utilizou o índice VERT como método de separação dos padrões faciais. Dentro desta mesma linha, Braga Reis et al. ${ }^{5}$ selecionaram, através de fotografias de perfil, indivíduos Padrão I e aferiram os valores das bases apicais. Estes valores foram maiores que os valores médios do grupo total aqui encontrado, o que era de se esperar, visto a amostra utilizada nesta pesquisa ter componentes tanto mesofaciais, quanto dolicofaciais e braquifaciais.

Como bem explicado por Delaire ${ }^{11}$, em 1976, a base do crânio é o alicerce sobre o qual se edifica a face humana e, por isso, sua anatomia condiciona a posição e o tamanho dos maxilares, influenciando a arquitetura facial. Os propósitos aqui defendidos e as conclusões obtidas reforçam a tese da importância da relação da anatomia craniana com os maxilares e a face, e da importância das normas serem individualizadas de acordo com o padrão facial do indivíduo, visto que foi obtida correlação estatisticamente significante da variável N.S.Ba com o índice indicativo do padrão facial (VERT) e com as medidas angulares das bases apicais.

Tendo em vista os resultados encontrados, seria de suma importância a realização de novas pesquisas, de novos estudos cefalométricos para avaliar o comportamento destas e de outras variáveis relativas à conformação craniofacial, contribuindo, assim, para o conhecimento e engrandecimento de nossa ciência, quantificando cada vez mais as variações numéricas que traduzem a normalidade facial.

\section{CONCLUSÃO}

Considerando-se a metodologia empregada e baseando-se nos resultados obtidos no decorrer desta investigação, concluiu-se que:

\section{Quanto à correlação entre a anatomia da base craniana e o padrão facial}

O comprimento da base anterior do crânio (S-N) e o ângulo S-N.Po-Or não apresentaram correlação estatisticamente significante com o índice indicativo do padrão facial (VERT). A deflexão da base do crânio (N.S.Ba) apresentou correlação positiva (quanto maior N.S.Ba maior o VERT) estatisticamente significante com o indice indicativo do padrão facial (VERT).

\section{Quanto à correlação entre a anatomia da base craniana e as bases apicais}

O comprimento da base anterior do crânio (S-N) não apresentou correlação estatisticamente significante com nenhuma das variáveis das bases apicais estudadas. Já os ângulos S-N. 
Po-Or e N.S.Ba apresentaram correlação negativa (quanto maior S-N.Po-Or e N.S.Ba menor o S.N.A e S.N.B) estatisticamente significante com as variáveis S.N.A e S.N.B, porém não apresenta- ram correlação estatisticamente significante com a variável A.N.B.

\title{
Cephalometric study of the cranial base anatomy correlation with the facial pattern and apical bases
}

\begin{abstract}
Aim: This cross-sectional study was conducted with the object of making a cephalometric evaluation of the cranial base anatomy correlation with the facial pattern and apical bases. Methods: 88 lateral teleradiographies of young white Brazilian with mean age of 10.3 years were used. The Ricketts VERT index was used to determine the facial pattern, and the sample was distributed as follows: 37 in group M (mesofacial), 34 in group D (dolicofacial) and 17 in group B (brachyfacial). The anatomic drawing, demarcation of points, line and plane tracings, and linear and angular measurement gauging were done manually. The cranial base measurements used were S-N, N.S.Ba and S-N.Po-Or and the apical base measurements were S.N.A, S.N.B and A.N.B. Results and Conclusion: It was concluded that, in the correlation between the cranial base and the facial pattern, there was significance between the variable N.S.Ba and the VERT Index. In the correlation between the cranial base and the apical bases, there was significance between N.S.Ba and the variables S.N.A and S.N.B, and between S-N.Po-Or and the variables S.N.A and S.N.B.
\end{abstract}

Key words: Facial pattern. Cranial base. Cephalometry.

\section{REFERÊNCIAS}

1. BHAT, M.; ENLOW, D. H. Facial variations related to head form type. Angle Orthod., Appleton, v. 55, no. 4, p. 269-280, 1985.

2. BJORK, A. Cranial base development. Am. J. Orthod. Dentofacial Orthop., St. Louis, v. 41, no. 3, p. 198-225, 1955.

3. BJORK, A. Relaciones de los maxilares com el cráneo. In: LUNDSTRON, A. Introduccion a la Ortodoncia. Buenos Aires: Mundi, 1971. p. 104-140.

4. BRODIE, A. G. The behavior of cranial base and its components as reveled by serial cephalometric roentgenograms. Angle Orthod., Appleton, v. 25, no. 3, p. $148-160,1955$.

5. BRAGA REIS, A. S. et al. Características cefalométricas dos indivíduos Padrão I. Rev. Dental Press Ortodon. Ortop. Facial, Maringá, v. 10, n. 1, p. 67-78, 2005.

6. CARDOSO, M. A. Características cefalométricas do padrão face longa. Rev. Dental Press Ortodon. Ortop. Facial, Maringá, v. 10, n. 2, p. 29-43, 2005.

7. CARREIRO, L. S. Influência da divergência facial, da deflexão e do comprimento da base anterior do crânio, sobre as bases apicais e os dentes, em jovens leucodermas brasileiros com oclusão normal, utilizando-se a análise de McNamara Jr. Ortodontia, São Paulo, v. 29, n. 3, p. 4-13, 1996.

8. CHRISTIE, T. E. Cephalometric patterns of adults with normal occlusion. Angle Orthod., Appleton, v. 47, n. 2, p. 128-135, 1977.
9. COTRIM- FERREIRA, F. A. Estudo cefalométrico do ângulo násio-sela-básio e da proporção entre as bases cranianas anterior e posterior, relacionados aos tipos faciais de Ricketts. 1993. Dissertação (Mestrado)-Faculdade de Odontologia, Universidade de São Paulo, São Paulo, 1993.

10. COTRIM-FERREIRA, F. A. Estudo cefalométrico radiográfico longitudinal de algumas medidas angulares e lineares da base craniana, dos tipos morfológicos faciais e suas possíveis correlações. 1999. Tese (Doutorado)-Faculdade de Possiveis correlações. 1999. Tese (Doutorado)-Faculdade de

11. DELAIRE, J. Curso de cirurgia y ortopedia dento maxilar. División publicaciones y ediciones de la Universidad de la República, Montevideo, 1976.

12. ENLOW, D. H.; KURODA, T.; LEWIS, A. B. The morphological and morphogenetic basis for craniofacial form and pattern. Angle Orthod., Appleton, v. 41, no. 3, p. 161-188, 1971.

13. ERDOGLIJA, L. J. Dynamics of the cranial base angle changes during the second trimester of the normal intrauterine growth and development. B. Bilt. Udruz. Ortodonata Jugosl., Beograd, v. 22, no. 1, p. 7-14, 1989.

14. ISAACSON, J. R. et al. Extreme variation in vertical face growth and associated in skeletal and dental relations. Angle Orthod., Appleton, v. 41, no. 3, p. 219-129, 1971.

15. JACOBSON, A. Radiographic cephalometry: from basics to videoimaging. Chicago: Quintessence, 1995. 
16. JARVINEM, S. Floating norms for the ANB angle as guidance for clinical considerations. Am. J. Orthod. Dentofacial Orthop., St. Louis, v. 90, no. 5, p. 383-387, 1986.

17. LEWIS, A. B.; ROCHE, A. F. The sadle Angle: constancy or change. Angle Orthod., Appleton, v. 47, no. 1, p. 46-54, 1977

18. LEWIS, A. B.; ROCHE, A. F.; WAGNER, B. Pubertal spurts in cranial base and mandible. Comparisons within individuals. Angle Orthod., Appleton, v. 55, no. 1, p. 17-30, 1985.

19. MCNAMARA JR. J. A A method of cephalometric evaluation. Am. J. Orthod. Dentofacial Orthop., St. Louis, v. 86, no. 6, p. $449-469,1984$

20. METZDORF, D. F. W. A cephalometric study of cranial, mandibular and lower incisor morphology in adult face. Angle Orthod., Appleton, v. 47, no. 4, p. 288-292, 1977.

21. REZK, E. R. The stability of the cranial base. Rev. Soc. Colomb. Orthod., [s.I.], v. 1, n. 4, p. 5-14, 1966.

22. RICKETTS, R. M. The cranial base and soft structures in cleft palate speech and breathing. Plastic. Reconstr. Surg., [s. I.], v. 14, p. 47-61, 1954.

23. RICKETTS, R. M. Planning treatment on the basis of the facial pattern and an estimate of its growth. Angle Orthod. Appleton, v. 27, no. 1, p. 4-37, 1957

24. RICKETTS, R. M. A foundation for cephalometric communication. Am. J. Orthod. Dentofacial Orthop., St Louis, v. 46, no. 5, p. 330-357, 1960

25. RICKETTS, R. M. Provocations and perceptions in craniofacial orthopedics. R.M.O., Denver, v. 1, no. 1, p. 10-35, 1989.
26. RAMOS, A. L. Influência da deflexão da base do crânio no posicionamento dentário e das bases apicais, em jovens brasileiros com oclusão normal. Ortodontia, São Paulo, v. 30 n. 4, p. 45-50, 1997

27. SAKIMA, M. T. Avaliação cefalométrica da relação entre a deflexão da base do crânio e a posição ântero-posterior $e$ vertical da maxila e da mandíbula em pacientes Classe II, $1^{\text {a }}$ divisão de Angle, com diferentes padrões craniofaciais. 1992. Dissertação (Mestrado)-Faculdade de Odontologia de Araraquara, Universidade Estadual Paulista Júlio de Mesquita Filho, Araraquara, 1992.

28. SIELAFF, R. The angulation of the base of the skull - a determining factor for facial skeletal development? The significance of the angulation of the base of the skull. Dtsch. Zahn. Mund. Kieferheilkd. Zentralbl., Leipzig, v. 79, no. 5, p. 365-373, 1991

29. STEINER, C. C. Cephalometrics in clinical practice. Angle Orthod., Appleton, v. 29, no. 1, p. 8-29, 1959.

30. TWEED, C. H. Clinical Orthodontics. St. Louis: Mosby 1966

31. VARGAS, J. N. Avaliação comparativa entre a linha selanásio e o plano horizontal de Frankfurt como parâmetros para o diagnóstico da posição ântero-posterior e vertical das bases ósseas, em jovens brasileiros leucodermas com más-oclusões de Classe I e II de Angle. 1998. Dissertação (Mestrado)-Faculdade de Odontologia de Bauru, Universidade de São Paulo, Bauru, 1998
Endereço para correspondência

Marcelo Calvo de Araújo

Rua Prof. Miguel Couto n 389/ apt: 1504 - Icara

CEP: 24.230-240 - Niterói/RJ

E-mail: marcelo@smileortodontia.com.br 